\title{
Eye-movement-contingent display changes are not compromised by flicker and phosphor persistence
}

\author{
ALBRECHT W. INHOFF, MATHEW STARR, and WEIMIN LIU \\ State University of New York, Binghamton, New York \\ and \\ JLAN WANG \\ Hangzhou University, Hangzhou, China
}

\begin{abstract}
The eye-movement-contingent display change technique has been used in a large number of studies to control the visibility of text when eye movements are monitored. Recently, the validity of the method has been questioned. O'Regan (1990) argued that technical imperfections in the implementation of the display change, rather than manipulation of visible text, account for some of the reported effects. This view was tested in the present study by manipulating characteristics of the display change and the linguistic task. Consistent with prior results, reading was hampered when eye-movement-contingent display changes were used to mask segments of text prior to their fixation. However, these costs were unrelated to the quality of the implemented display change and appear to have derived solely from the masking of useful text.
\end{abstract}

In a typical experiment that yields oculomotor data, the experimenter controls the characteristics of the visible stimulus, and the participant controls the encoding of information by determining where to move the eyes and for how long to look (fixate). Effects of stimulus characteristics on oculomotor activity, comprising fixations and jump-like movements of the eyes (saccades), can then be used to make inferences about on-line cognitive processes and cognitive architectures. This provides a number of methodological advantages over more conventional paradigms (Just \& Carpenter, 1980, 1987; Rayner, 1978; Rayner \& Pollatsek, 1989). Oculomotor activity generally constitutes an integral part of the experimental task, and its continuous measurement obviates the need for the execution of the discrete, extraneous responses required in most other experimental tasks. This reduces the risk that task- and response-specific demands might exaggerate, attenuate, or alter experimental effects (Magliano, Graesser, Eymard, Haberland, \& Gholson, 1993).

The methodological promise of oculomotor measures has resulted in a dramatic increase of usage in recent years. Until approximately 15 years ago, they were used by a relatively small number of researchers who studied perceptual processes during reading and picture perception. Today, they are used across a broad range of domains, including linguistic analyses during reading (see Just \&

This research was supported by NIMH Grant 503870 and by the Center for Cognitive and Psycholinguistic Studies at SUNY Binghamton. We would like to thank Keith Rayner and Robert Crowder for their helpful comments. Correspondence should be addressed to A. W. Inhoff, Department of Psychology, State University of New York, Binghamton, NY 13902-6000 (e-mail: inhoff@binghamton.edu).
Carpenter, 1987, and Rayner \& Pollatsek, 1989, for reviews); retrieval and inference processes (e.g., Garrod, Freudenthal, \& Boyle, 1994); picture/scene perception (e.g., Rayner \& Pollatsek, 1992); the encoding and comprehension of visual charts and figures (e.g., Hegarty, 1992); and the recognition of auditory speech (Tanenhaus, Spivey-Knowlton, Eberhard, \& Sedivy, 1995).

As noted, the experimenter typically selects the characteristics of the visible stimulus-for example, a segment of text - and the participant determines how and when it is fixated. This entails some loss of experimental control, since the participant-rather than the experimenter-determines how and when information is encoded. Eye-movement-contingent display change techniques (McConkie \& Rayner, 1975; Rayner, 1975) avoid this shortcoming by returning control over temporal, spatial, and linguistic characteristics of visible text to the experimenter.

In McConkie and Rayner's (1975) version of the technique, usually referred to as the eye-movement-contingent window method, the visibility of text is controlled by moving a predefined area (window) of legible text in synchrony with a reader's eyes. Degraded or illegible text is shown outside the window. In Rayner's (1975) version of the technique, generally referred to as the boundary method, display changes are applied to a preselected target location. Prior to the fixation of this location, a stimulus that may be related to the target word is displayed. When the eyes cross a prespecified spatial area (the boundary) on their way to the target location, usually consisting of the blank space preceding the target location, the target word is displayed. Target viewing durations, as a function of the previously visible information, can then be used to determine the types of information that were obtained prior to the crossing of the target's boundary. 
The window method has been used to investigate perceptual processes during the reading of English, Hebrew, Japanese, and Chinese texts (e.g., Inhoff \& Liu, 1998; Osaka, 1992; Pollatsek, Bolozky, Well, \& Rayner, 1981; Rayner, Well, Pollatsek, \& Bertera, 1982) and during copy typing (Inhoff, Briihl, Bohemier, \& Wang, 1992; Inhoff \& Wang, 1992). Results from these studies converge in showing that, irrespective of script type, skilled reading and typing require the acquisition of useful information from the fixated (foveal) word and from subsequent (parafoveal) words in the text. Experiments using the boundary technique delineated the representation of parafoveally acquired information. They showed that readers obtain useful letter-level, phonological, and lexical information from the parafovea and that the computation of parafoveal word representations is a function of contextual constraints. For instance, a word's viewing duration has been shown to be shorter when parafoveal preview revealed similar letters than when it revealed dissimilar letters (Rayner, 1975; Rayner et al., 1982), when the preview was homophonic rather than nonhomophonic (Pollatsek, Lesch, Morris, \& Rayner, 1992), when it consisted of a high-frequency word rather than a lowfrequency word (Inhoff \& Rayner, 1986), and when contextual constraints were high rather than low (Balota, Pollatsek, \& Rayner, 1985).

However, the two eye-movement-contingent display change techniques have been subjected to methodological criticism. In his assessment of the method, O'Regan (1990) noted that a masked parafoveal stimulus may have been visible for $10 \mathrm{msec}$ or more after the saccade to it was completed; the flicker resulting from the replacement of the masking stimulus with the to-be-implemented target stimulus during a fixation "may act as a warning signal which modifies saccadic latency" (i.e., fixation duration; p. 404). Flicker, rather than parafoveal masking of useful text, could thus account for window- and boundary-related effects. Specifically, the magnitude of flicker should increase with decreases in window size, hence accounting for any increase in fixation durations with decreases in window size. Flicker should also be more noticeable when a parafoveally previewed and subsequently fixated stimulus are visually dissimilar than when they are similar, hence accounting for orthographyrelated effects. Moreover, flicker could also cause an overreliance on parafoveally obtained linguistic information. Specifically, disruption of visuospatial analyses during fixation onset may delay the activation of phonological and lexical representation of fixated text, possibly leading to an increased usage of previously (i.e., parafoveally) obtained linguistic information during the fixation.

O'Regan (1990) also argued that eye-movementcontingent display changes can be compromised by phosphor persistence. CRT phosphors may not be completely faded when the display change is implemented, and residual traces from the replaced masking stimulus may erode the letter contrasts of the subsequently implemented stimulus. For instance, when the masking char- acter $o$ is replaced with the target character $c$, phosphoric afterglow could bridge the target character's open gap, hampering its identification. Again, effects of phosphor persistence should be particularly noticeable when the window of legible text is small and when computation of visuospatial codes for fixated text is abandoned in favor of more abstract linguistic codes derived from previously visible parafoveal information.

Yet, virtually no experimental data are available to judge the merits of O'Regan's (1990) hypothesis. To examine the validity of O'Regan's concerns directly, we manipulated the occurrence of flicker and of phosphoric persistence. According to O'Regan, these manipulations should define - or at least contribute to- the magnitude of eye-movement-contingent display change effects during reading.

\section{EXPERIMENT 1}

\section{Method}

Participants. Eight undergraduates from the State University of New York at Binghamton participated for experimental course credit.

Apparatus. The hardware setup was identical to the setup of prior studies, which may be consulted for details (e.g., Vitu, O'Regan, Inhoff, \& Topolski, 1995). Eye movements were recorded via an SRI eyetracking system (Crane \& Steele, 1985). The system has an absolute horizontal resolution of approximately $1^{\prime}$ of arc and its output was linear over the horizontal tracking range. Fixation locations were determined every $2 \mathrm{msec}$ and were used to compute oculomotor measures.

Materials. Eight passages taken from a larger set of passages (Vitu et al., 1995) were read. All were relatively easy to comprehend and contained 80-100 words.

Procedure. We adopted Vitu et al.'s (1995) passage reading and letter detection procedure. The experiment began with a calibration of the oculomotor system. The calibration was considered successful whenever it yielded a tracking accuracy of $\leq 1$ character space. After this, a practice passage and the experimental passages of text were shown. Only one line of a passage was visible at a time, and passage reading progressed line by line. The reader controlled line onsets and offsets by pressing a mouse key button. The accuracy of the oculomotor tracking procedure was checked in between the presentation of successive lines of text.

Participants were asked to read for comprehension in one task condition and to read for comprehension and to detect the presence of a critical letter in another task condition. The character $c$ was used as the to-be-detected (critical) letter and each passage contained four critical items. Detection was signaled by depressing a key on a QWERTY keyboard (although the software setup did not monitor keyboard entries). The detection task was added to the comprehension task as of means of increasing readers' reliance on low-level (featural) codes that should be particularly susceptible to flicker effects.

All passages were read in two viewing conditions. In the no-mask condition, the full line of text was visible during each fixation, as occurs during normal reading. In the parafoveal-masking condition, each letter of each word to the right of fixation was replaced by a visually dissimilar letter, and each word became fully visible when it was fixated. This was accomplished by defining $1 / 10$ of the blank space that followed the last character of each word as the display change boundary for the next word in the text (the horizontal $x$ value of the eye-tracker output was monitored, and whenever its output was equal to or greater than the boundary's horizontal $x$ value, the display for the next word was implemented). Software routines were used that determined to-be-displayed text within the 2-msec fixation location sampling cycle that fulfilled the display change criterion

Implementation of the display change was, however, not immediate. It was controlled by varying the speed of the vertical refresh rate - that is, the speed with which a required display change could be imple- 
mented on the CRT's next refresh cycle. Four rates were selected that refreshed at $60,72,120$, and $200 \mathrm{~Hz},{ }^{1}$ resulting in implementation delays of approximately $16,14,8$, and $5 \mathrm{msec}$, respectively. Flicker was assumed to have occurred whenever the display change implementation delay exceeded the interval in between the crossing of a word's boundary and the onset of its subsequent fixation. It was expected to occur more often when implementation lags were long than when they were short.

Design and data analyses. Two lists (A and B) of eight passages were created. On each list, half of the lines of a passage were fully visible during reading (control condition); parafoveal words were masked during the reading of the remaining lines (masking conditions), with control and masking lines being ordered randomly. Both lists were identical except that fully visible and masked lines of text on List A were replaced with masked and fully visible lines on List B. On each list, two passages were shown in each of the four horizontal refresh rate conditions One of the two passages in each refresh rate condition was read for meaning, and the other was read for meaning with concurrent letter detection. List assignment was counterbalanced across successive participants.

Each participant read one list, so that refresh rate (4), task type (2), and eye-movement-contingent masking (2) were manipulated within subjects. Effects of the three independent variables were sought in three commonly used measures: gaze durations, first-fixation durations, and interword saccade size. Gaze durations measured the cumulated viewing duration of a word prior to the fixation of another word (they included intraword refixation durations but not the time spent moving the eyes during intraword saccades). First-fixation durations measured the duration of the first target fixation, irrespective of whether the word was refixated or not, and interword saccades measured the size of saccades (in character spaces) that led to a first-fixation duration. All words containing three or more letters were considered targets.

Gazes and first-fixation durations of $1,000 \mathrm{msec}$ or more were excluded from analyses to eliminate outliers (approximately $2 \%$ of the data). We also excluded all instances in which a first fixation fell on the blank space preceding a target, when a first fixation was preceded by an extremely large saccade extending across 20 character spaces or more, and when a first fixation was preceded by a left-directed regressive saccade (approximately $5 \%$ of the data). Fixation durations of less than $100 \mathrm{msec}$ were not included because they do not appear to be sensitive to effects of parafoveal masking (Inhoff, Topolski, \& Wang, 1992; McConkie, Underwood, Zola, \& Wolverton, 1985). Gaze durations, firstfixation durations, and saccade size, each comprising 4,850 measures, were analyzed with a 2 (parafoveal masking) $\times 2$ (task type) $\times 4$ (refresh rate) within-subjects analysis of variance (ANOVA)

\section{Results}

Effects of eye-movement-contingent display changes as a function of refresh rate, parafoveal visibility of text, and experimental task are shown in Table 1. Consistent with previously reported results, analyses of gaze durations showed that masking of parafoveally visible text incurred costs, amounting to approximately $66 \mathrm{msec}[F(1,7)=$ $26.73, p<.001]$, and that reading of text plus letter detection was more demanding than mere reading for comprehension [a 33-msec effect; $F(1,7)=12.98, p<.01]$.

In violation of the flicker hypothesis, examination of gaze durations also revealed virtually identical masking effects for slow and fast refresh rates, irrespective of whether the task entailed reading of text or reading plus letter detection. Specifically, masking effects for the two slow $(60 / 72 \mathrm{~Hz})$ and fast $(120 / 200 \mathrm{~Hz})$ refresh conditions amounted to 65 and $63 \mathrm{msec}$, respectively, in the reading task and 71 and $66 \mathrm{msec}$ in the reading plus letter detection task. The two-way interaction of refresh rate and masking and the three-way interaction of refresh rate, masking, and reading task were negligible (both $F \mathrm{~s}<1$ ).

Striking similarities of masking effects across refresh rates were also evident in the first-fixation durations and interword saccades, also shown in Table 1. The main effects of masking and task type were again robust in firstfixation durations $[F(1,7)=19.95, p<.005 ; F(1,7)=$

Table 1

Mean Gaze Durations, First-Fixation Durations,

Intertarget Saccade Size (in Milliseconds), and

Standard Errors (SE) as a Function of Refresh Rate, Masking Condition, and Reading Type

\begin{tabular}{|c|c|c|c|c|c|c|c|c|c|}
\hline \multirow[b]{3}{*}{ Refresh Rate } & \multicolumn{4}{|c|}{ Parafoveal Masking } & \multicolumn{4}{|c|}{ No Parafoveal Masking } & \multirow[b]{3}{*}{$M$} \\
\hline & \multicolumn{2}{|c|}{ Read/Detect } & \multicolumn{2}{|c|}{ Read Only } & \multicolumn{2}{|c|}{ Read/Detect } & \multicolumn{2}{|c|}{ Read Only } & \\
\hline & $M$ & $S E$ & $M$ & $S E$ & $M$ & $\overline{S E}$ & $M$ & $S E$ & \\
\hline \multicolumn{10}{|c|}{ Gaze } \\
\hline 200 & 385 & 23 & 321 & 26 & 322 & 18 & 262 & 18 & 323 \\
\hline 120 & 370 & 31 & 348 & 19 & 291 & 18 & 276 & 22 & 321 \\
\hline 72 & 380 & 31 & 359 & 23 & 306 & 25 & 285 & 21 & 333 \\
\hline 60 & 376 & 31 & $34 !$ & 18 & 320 & 28 & 289 & 24 & 332 \\
\hline$M$ & 378 & & 342 & & 309 & & 278 & & \\
\hline \multicolumn{10}{|c|}{ First-Fixation Durations } \\
\hline 200 & 289 & 10 & 273 & 16 & 265 & 13 & 230 & 7 & 264 \\
\hline 120 & 301 & 27 & 290 & 11 & 242 & 14 & 234 & 11 & 267 \\
\hline 72 & 294 & 19 & 290 & 16 & 266 & 16 & 243 & 13 & 273 \\
\hline 60 & 311 & 17 & 296 & 5 & 266 & 18 & 242 & 11 & 279 \\
\hline$M$ & 298 & & 237 & & 259 & & 237 & & \\
\hline \multicolumn{10}{|c|}{ Saccade Size } \\
\hline 200 & 7.6 & .5 & 8.3 & .6 & 8.4 & .4 & 9.2 & .5 & 8.4 \\
\hline 120 & 7.3 & .5 & 7.2 & .5 & 8.3 & .6 & 9 & .4 & 8 \\
\hline 72 & 7.4 & .5 & 8.1 & .6 & 8.4 & .5 & 9.3 & .4 & 8.3 \\
\hline 60 & 7.8 & .9 & 8.6 & .9 & 8.5 & .7 & 9.1 & .6 & 8.5 \\
\hline$M$ & 7.6 & & 8.1 & & 8.4 & & 9.2 & & \\
\hline
\end{tabular}


$6.62, p<.05$, respectively $]$ and saccade size $[F(1,7)=$ $36.5, p<.001 ; F(1,7)=9.48, p<.025]$. Furthermore, refresh rate did not interact with masking in either the first-fixation durations $[F(1,7)=1.52, p>.23]$ or in the saccade size data $(F<1)$. The three-way interaction of refresh rate, masking, and reading type was also negligible in both sets of data $(F<1)$. None of the remaining effects approached significance. ${ }^{2}$

\section{Discussion}

Replicating prior results, the masking of parafoveally visible text hampered reading, increasing gaze durations and first-fixation durations, and decreasing saccade size. However, these costs cannot be attributed to the occurrence of flicker. In violation of the flicker account, effects of masking were virtually identical in the slowest and fastest refresh rate conditions, although flicker should have been more likely when slow refresh rates were used. Moreover, slow refresh rates did not hamper letter detection, even though feature- and letter-level analyses should have been disrupted by flicker.

Effects of parafoveal masking may have been independent of effects of refresh rate because flicker occurred too infrequently in each of the four refresh rate conditions. To ascertain this possibility, we estimated flicker frequencies for the two extreme refresh rate conditions ( 60 and $200 \mathrm{~Hz}$ ) for 1 reader with nearly median gaze durations. This estimate indicated that flicker followed approximately $16 \%$ of fixation onsets in the $60-\mathrm{Hz}$ condition and $<1 \%$ in the $200-\mathrm{Hz}$ condition. Occasional flicker thus did occur in the slow refresh rate conditions but did not interact with masking.

It is possible that the oculomotor system accommodates flicker during the beginning few milliseconds of a fixation by considering it part of the visuospatial "noise" that occurs during the onset of a fixation. During this period, the two eyes accommodate, and the lens appears to move briefly in the opposite direction of the saccade (Deubel, 1995).

\section{EXPERIMENT 2}

Experiment 2 examined the "erosion-of-contrast" hypothesis by comparing effects of display changes that were implemented on a phosphor-based CRT and an electroluminescent (EL) panel. In contrast to phosphors, EL gases decay instantaneously and should not suffer any erosion of contrast. Two eye-movement-contingent display change conditions were created. In one, a parafoveally visible target word was masked by replacing each of its letters with a visually dissimilar symbol. In the second condition, a parafoveally visible target was also replaced with visually dissimilar characters, except for its fourth character, which was replaced with a blank space. Hence, there should be no erosion of contrast for the target's fourth character location. No masking occurred in the control condition. According to the erosion-of-contrast hypothesis, the parafoveal masking of target words and their eyemovement-contingent unmasking should be more disruptive when the display change is implemented on a phosphor-based CRT than when it is implemented on an EL panel. Furthermore, effects of display changes on the CRT screen should be larger when all characters of the target are replaced than when a subset is replaced.

\footnotetext{
Method

Participants. Thirty undergraduate students at the State University of New York at Binghamton participated in the experiment. They re-
}

ceived either experimental course credit or pay. All were native speakers of English and naive about the purpose of the experiment.

Materials and viewing conditions. The materials consisted of 96 unrelated sentences, each of which contained 6 to 11 word constituents, including 1 target word that occupied neither sentence-beginning nor sentence-ending location. All targets were nouns containing seven to nine characters. The target of each sentence was shown in one of three viewing conditions. It was continuously visible in the control condition, as occurs in normal reading. It was masked in two parafoveal-masking conditions. In the full-erosion condition, all characters of the target were masked until the eyes crossed the target's boundary. For instance, the parafoveal target "movement" would be masked by the character sequence vinquizp until the eyes moved onto the target area, after which the mask was removed. In the partial-erosion condition, all characters of the target except its fourth character were masked with dissimilar characters. The fourth character was replaced with a blank space. For instance, the target "movement" was masked by the character sequence vin uizp until it was fixated. As in Experiment 1 , the beginning $1 / 10$ of the blank space preceding each target constituted its boundary.

Apparatus. Half of the participants viewed text on a Sharp flat-panel EL display that was controlled by a Yamaha VGA card and a custommade display driver (developed by Yamaha Corp.). The EL measured 10.5 in. diagonally. It used a vertical refresh rate of $120 \mathrm{~Hz}$ and presented text in light orange on a black background. EL gases decay immediately, thus avoiding the minute brightness trails of decaying phosphors. The distance between readers' eyes and the panel was set at $45 \mathrm{~cm}$; at this viewing distance, each character of text subtended approximately $.25^{\circ}$ of visual angle. The remaining participants viewed text using a CRT that was equipped with a sulfide-copper P-22 color phosphor, the green component of which decays to $2 \%$ of its peak intensity within $360 \mu \mathrm{sec}$. A display change implementation rate of $200 \mathrm{~Hz}$ was selected because this extremely rapid rate of screen refresh should exaggerate any potential effect of contrast reduction.

Procedure, scoring design, and data analyses. Similar to Experiment 1 , one line of text, now comprising one unrelated sentence, was shown at a time, and the accuracy of eye tracking was checked after each sentence was read. The selection of data and the definition of dependent measures were also identical in Experiments 1 and 2. Three lists of identical sentences were created so that each target could be shown in a different target (pre)viewing condition. Half of the readers viewed text on a CRT screen, and the remaining readers viewed text on the EL panel. For each display type, list assignment was counterbalanced across 3 successive readers. All data were analyzed with an ANOVA; the between-subjects factor was display (2), and the within-subjects factor was target preview (3).

\section{Results}

Gaze durations, first-fixation durations, and interword saccades as a function of display type and target preview are shown in Table 2. Again, the gaze durations on target words were shorter when the target was visible in the parafovea prior to its fixation than when all or part of its letters had been masked, with corresponding gaze durations of 285,351 , and $334 \mathrm{msec}[F(2,56)=30.22, p<$ $.001]$. In violation of the erosion-of-contrast hypothesis, effects of masking were slightly more detrimental for EL displays than for CRT displays, with the difference between the control and full-erosion conditions amounting to 57 and $42 \mathrm{msec}$, respectively, although the statistical interaction of parafoveal preview and display panel was negligible $(F<1)$. Moreover, in direct contradiction to the erosion-of-contrast hypothesis, gazes on CRT screen text were $15 \mathrm{msec}$ shorter in the full-erosion condition than in the partial-erosion condition.

Effects of display panel were also negligible in the firstfixation durations and saccade size data (both $F \mathrm{~s}<1$ ). 
Table 2

Oculomotor Activity (in Milliseconds) and

Standard Errors (SE) During Target Reading in Experiment 2

\begin{tabular}{|c|c|c|c|c|c|c|c|c|c|c|c|c|}
\hline \multirow[b]{4}{*}{ Erosion } & \multicolumn{12}{|c|}{ Display Type } \\
\hline & \multicolumn{4}{|c|}{ Control } & \multicolumn{4}{|c|}{ Partial } & \multicolumn{4}{|c|}{ Full } \\
\hline & \multicolumn{2}{|c|}{ CRT } & \multicolumn{2}{|c|}{ EL } & \multicolumn{2}{|c|}{ CRT } & \multicolumn{2}{|c|}{ EL } & \multicolumn{2}{|c|}{ CRT } & \multicolumn{2}{|c|}{ EL } \\
\hline & $M$ & $S E$ & $M$ & $S E$ & $M$ & $S E$ & $M$ & $S E$ & $M$ & $\overline{S E}$ & $M$ & $\overline{S E}$ \\
\hline Gaze durations & 289 & 15 & 280 & 7 & 346 & 12 & 356 & 16 & 331 & 11 & 337 & $\overline{11}$ \\
\hline First-fixation durations & 257 & 11 & 256 & 8 & 279 & 9 & 300 & 11 & 285 & 9 & 292 & 8 \\
\hline Saccade size & 8.4 & .3 & 9.0 & .4 & 8.5 & .4 & 8.6 & .4 & 8.4 & .3 & 8.5 & .4 \\
\hline
\end{tabular}

Note-EL, electroluminescent.

Parafoveal masking increased first fixations and yielded slightly shorter saccades; the corresponding effects were reliable in first-fixation durations $[F(1,56)=19.66, p<$ $.001]$, but not in the saccade data $[F(2,56)=1.76, p>$ $.25]$. The interaction of display type, predicted by the erosion-of-contrast hypothesis, again failed to approach significance [first-fixation durations, $F<1$; saccade size, $F(2,56)=2.11, p>.1]$.

\section{Discussion}

The results of Experiment 2 provide compelling evidence against the erosion-of-contrast hypothesis. In direct opposition to its predictions, effects of eye-movement-contingent masking were slightly smaller when implemented on a CRT screen than when implemented on an EL panel, and full erosion of contrast was slightly less detrimental than partial erosion of contrast. The decay of "fast" phosphors, including the green component of the commonly used $\mathrm{p} 22$ compound, is thus rapid enough to prevent any erosion of contrast.

\section{CONCLUSION}

Eye-movement-contingent display change techniques can be used to control the visibility of text under ecologically valid conditions. Unless atypically slow refresh rates or relatively slow phosphors are used, results based on the use of the techniques are compromised neither by flicker nor by an erosion of character contrasts. A reappraisal of previously reported effects is unwarranted.

\section{REFERENCES}

Balota, D. A., Pollatsek, A., \& Rayner, K. (1985). The interaction of contextual constraints and parafoveal visual information in reading. Cognitive Psychology, 17, 364-390.

Crane, H. D., \& Steele, C. M. (1985). Generation-V dual-Purkinjeimage eye tracker. Applied Optics, 24, 527-537.

Deubel, H. (1995). Fourth Purkinje image signals reveal eye-lens deviations and retinal image distortions during saccades. Vision $R e$ search, 35, 529-538.

Garrod, S., Freudenthal, D., \& Boyle, E. (1994). The role of different types of anaphors in the on-line resolution of sentences in a discourse. Journal of Memory \& Language, 33, 39-68.

HEgARTY, M. (1992). The mechanics of comprehension and the comprehension of mechanics. In K. Rayner (Ed.), Eye movements and visual cognition: Scene perception and reading (pp. 428-443). New York: Springer-Verlag.

INHOFF, A. W., BrilhL, D., BohEMIER, G., \& WANG, J. (1992). Eye-hand span and the coding of text during copytyping. Journal of Experimental Psychology: Learning, Memory, \& Cognition, 18, 298-306
INHOFF, A. W., \& LIU, W. (1998). The perceptual span and oculomotor activity during the reading of Chinese sentences. Journal of Experimental Psychology: Human Perception \& Performance, 24, 20-34.

INHOFF, A. W., \& RAYNER, K. (1986). Parafoveal word processing during eye fixations in reading: Effects of word frequency. Perception \& Psychophysics, 40, 431-439.

INHOFF, A. W., \& TOPOLSKI, R., \& WANG, J. (1992). Saccade programming during short duration fixations: An examination of copytyping, letter detection, and reading. Acta Psychologica, 87, 1-21.

INHOFF, A. W., \& WANG, J. W. (1992). The encoding of text, manual movement planning, and eye-hand coordination during copytyping. Journal of Experimental Psychology: Human Perception \& Performance, 18, 437-448.

Just, M. A., \& CARPENTER, P. A. (1980). A theory of reading: From eye fixations to comprehension. Psychological Review, 87, 329-354.

Just, M. A., \& CARPENTER, P. A. (1987). The psychology of reading and language comprehension. Newton, MA: Allyn \& Bacon.

Magliano, J. P., Graesser, A. C., Eymard, L. A., Haberland, K., \& GHOLSON, B. (1993). Locus of interpretive and interference processes during text comprehension: A comparison of gaze durations and reading times. Journal of Experimental Psychology: Learning, Memory, \& Cognition, 19, 704-709.

MCCONKIE, G. W., \& RAYNER, K. (1975). The span of the effective stimulus during a fixation in reading. Perception \& Psychophysics, 17, 578-586.

MCCONkie, G. W., Underwood, N. R., Zola, D., \& Wolverton, G. S. (1985). Some temporal characteristics of processing during reading. Journal of Experimental Psychology: Human Perception \& Performance, 11, 168-186.

O'Regan, J. K. (1990). Eye movements in reading. In E. Kowler (Ed.), Eye movements and their role in visual and cognitive processes: $R e$ views of oculomotor research (pp. 395-454). Amsterdam: Elsevier.

OSAKA, N. (1992). Size of saccade and fixation duration of eye movements during reading: Psychophysics of Japanese text processing. Journal of the Optical Society of America, 9, 5-13.

Pollatsek, A., Bolozky, S., Well, A. D., \& Rayner, K. (1981). Asymmetries in the perceptual span for Israeli readers. Brain \& Language, 14, 174-180.

Pollatsek, A., Lesch, M., Morris, R., \& Rayner, K. (1992). Phonological codes are used in integrating information across saccades in word identification and reading. Journal of Experimental Psychology: Human Perception \& Performance, 18, 148-162.

RAYNER, K. (1975). The perceptual span and peripheral cues in reading. Cognitive Psychology, 7, 65-81.

RAYNER, K. (1978). Eye movements in reading and information processing. Psychological Bulletin, 85, 618-660.

Rayner, K., \& Pollatsek, A. (1989). The psychology of reading. Englewood Cliffs, NJ: Prentice Hall.

Rayner, K., \& Pollatsek, A. (1992). Eye movements and scene perception. Canadian Journal of Experimental Psychology, 46, 342-376.

Rayner, K., Well, A. D., Pollatsek, A., \& Bertera, J. H. (1982). The availability of useful information to the right of fixation in reading. Perception \& Psychophysics, 31, 537-550. 
Tanenhaus, M. K., Spivey-Knowlton, M. J., Eberhard, K. M., \& SEDIVY, J. C. (1995). Integration of visual and linguistic information during spoken language comprehension. Science, 268, 1632-1634.

Vitu, F., O'Regan, J. K., Inhoff, A. W., \& Topolski, R. (1995). Mindless reading: Eye-movement characteristics are similar in scanning letter strings and reading texts. Perception \& Psychophysics, 57, 352-364.

measurements confirmed that all refresh rates were accurately implemented.

2. Gaze durations and first-fixation durations decreased slightly with increases in refresh rates. However, saccades also decreased slightly with increased refresh rates, suggesting that there could have been some tradeoff between measures.

\section{NOTES}

1. A photometer, attached to an oscilloscope, was used to measure the interval between successive brightness peaks on the CRT surface. These

(Manuscript received May 5, 1995;

revision accepted for publication September 12, 1997.) 